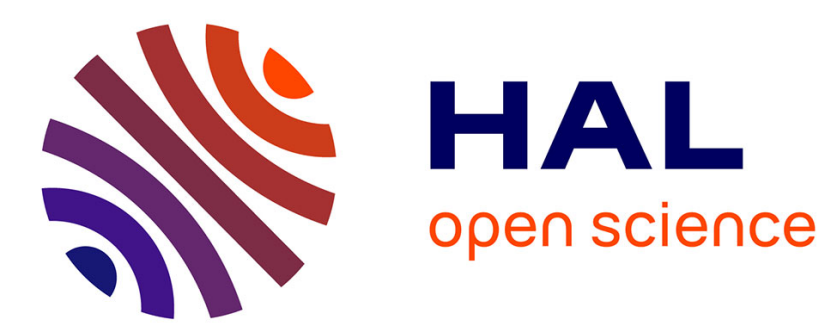

\title{
On the zero-dynamics of a class hybrid LTI systems: a geometric approach
}

Mattia Mattioni, Salvatore Monaco, Dorothée Normand-Cyrot

\section{To cite this version:}

Mattia Mattioni, Salvatore Monaco, Dorothée Normand-Cyrot. On the zero-dynamics of a class hybrid LTI systems: a geometric approach. IEEE Control Systems Letters, 2019, 3 (3), pp.703-708. 10.1109/lcsys.2019.2917589 . hal-02130672

\section{HAL Id: hal-02130672 \\ https://hal.science/hal-02130672}

Submitted on 16 May 2019

HAL is a multi-disciplinary open access archive for the deposit and dissemination of scientific research documents, whether they are published or not. The documents may come from teaching and research institutions in France or abroad, or from public or private research centers.
L'archive ouverte pluridisciplinaire HAL, est destinée au dépôt et à la diffusion de documents scientifiques de niveau recherche, publiés ou non, émanant des établissements d'enseignement et de recherche français ou étrangers, des laboratoires publics ou privés. 


\title{
On the zero-dynamics of a class hybrid LTI systems: a geometric approach
}

\author{
Mattia Mattioni ${ }^{1}$, Salvatore Monaco ${ }^{1}$ and Dorothée Normand-Cyrot ${ }^{2}$
}

\begin{abstract}
The paper deals with the geometric characterization of the zero-dynamics for linear time-invariant systems with aperiodic time-driven jumps. As the intuition suggests, it is given by the restriction of the feedback dynamics to the largest subspace over which the trajectories are constrained to ensure zero output. Such a dynamics is characterized by a subset of the flowing zeros and a subset of the zeros which can be fictitiously associated to the jumping dynamics.
\end{abstract}

Index Terms-Hybrid systems; Algebraic/geometric methods; Linear systems.

\section{INTRODUCTION}

Nowadays, growing attention is devoted toward dynamics characterized by the interaction of both continuous and discrete-time behaviors. Those kind of systems are referred to as hybrid systems and typically described by set inclusions and the interconnection of suitable discrete and continuoustime models characterizing the jumping and flowing evolutions which are governing, in a combined way, the overall dynamics [1]-[6]. Among these, hybrid systems with timedriven state jumps (or impulsive systems) are of paramount importance as they allow to fully describe, for example, cyber-physical systems or dynamical analog systems interconnected to digital devices (e.g., sampled-data systems) by simultaneously catching the heterogeneous behaviors acting over the overall system. When jumps are periodic in time, several works have been developed to address important control problems such as, for example, hybrid regulation [7], [8]. In those contributions, the notion of zero-dynamics has been shown to be, as in purely continuous or discrete-time systems, a fundamental issue that cannot be discarded [9][11]. Still, a complete characterization of this behavior has not been provided so that its analysis is typically lead (in a conservative way) to the corresponding purely continuous or discrete-time counterpart. The work in [12] represents a first attempt toward the characterization of zeros of a hybrid system via a suitably defined hybrid transfer function in a hybrid frequency domain. However, such an approach is quite involved and suffers from generalizability to a wider context as direct integration of the trajectories is needed for the definition of the zeros and thus motivating the periodic context. Moreover, because the transfer function consists of

Partially funded by the Université Franco-Italienne/Università ItaloFrancese through the Vinci Grant 2018.

${ }^{1}$ Dipartimento di Ingegneria Informatica, Automatica e Gestionale $A$. Ruberti (La Sapienza University of Rome); Via Ariosto 25, 00185 Rome, Italy \{mattia.mattioni, salvatore.monaco\}@uniroma1.it.

${ }^{2}$ Laboratoire de Signaux et Systèmes (L2S, CNRS); 3, Rue Joliot Curie, 91192, Gif-sur-Yvette, France dorothée. normand-cyrotel2s.centralesupelec.fr four components that are parametrized by two complex variables (for the flow and jump behaviors), explicitly exhibiting the zeros might not be easy in general.

In this paper, we address the problem of defining the zero-dynamics for linear hybrid systems with aperiodic timedriven jumps in the geometric framework developed in [13], [14] for general results and recently extended to this hybrid context in, for example, [15], [16]. In doing so, no knowledge of the jumping instants is assumed. In particular, instead of focusing on the definition of the zeros, we investigate the concept of zero-dynamics subspace allowing a revealing study of the hybrid zero-dynamics as defined by a suitable combination of the zero-dynamics corresponding to the flowing and jumping behaviors. Such an approach relies upon the definition of a suitable controlled-invariant subspace (the hybrid zero-subspace) that is contained into the nullspace of the output evolution and is, at the same time, invariant under the flow and jump dynamics. Connections with the hybrid zeros of the system are also established as particular subsets of couples of the flowing and fictious jumping zeros, associated to flowing and jumping dynamics when considered as purely continuous and discrete-time systems. In our context, the feedback laws inducing the zerodynamics is independent upon the jumping period sequence and requires no integration of the trajectories contrarily to what proposed in [12] or in the context of invariance at large in [15].

The remaining of the paper is organized as follows: in Section $[\mathrm{II}$ the class of systems under study is defined and the problem is settled. In Section III the hybrid zero-dynamics is characterized based on the definition of the hybrid zerosubspace which is controlled invariant under the hybrid system. Insights on the hybrid zero-dynamics are investigated in Section IV where the notion of hybrid zeros is also set. Some examples illustrate the results in Section $\mathrm{V}$ whereas conclusions and future perspectives are in Section VI.

\section{PRELIMINARIES AND THE CLASS OF SYSTEMS UNDER STUDY}

\section{A. Notations}

$\operatorname{Mat}_{\mathbb{R}}(n, m)$ defines the set of $n \times m$ matrices with real entries. Given a square matrix $A \in \operatorname{Mat}_{\mathbb{R}}(n, n)$ we denote by $|A|$ the determinant of $A$. The notation $\sigma(A)=\{\lambda \in$ $\mathbb{C}$ s.t. $|A-\lambda I|=0\}$ defines the spectrum of $A$. Given a matrix $B \in \operatorname{Mat}_{\mathbb{R}}(n, m)$, we say that $V=\operatorname{span}\left\{s_{1}, \ldots, s_{p}\right\} \subset$ $\mathbb{R}^{n}$ is $(A, B)$-invariant if, for all $s_{i} \in V, A s_{i} \in V+\operatorname{Im} B$ or, in short, $A V \subset V+\operatorname{Im} B$. Moreover, we say that $F$ is the 
friend of a controlled invariant subspace $V$ if $(A+B F) V \subset V$. We define the ordered set $\Pi(A, B, C):=\left\{V \subset \mathbb{R}^{n}\right.$ s.t. $A V \subset$ $V+\operatorname{Im} B$ and $V \subset \operatorname{ker} C\}$. With a slight abuse of notations, $\mathbb{R}_{n}^{\perp}$ denotes the dual space to $\mathbb{R}^{n}$ while $\Omega A$ denotes the subspace of $\mathbb{R}_{n}^{\perp}$ generated by the rows of $\omega A$ for $\omega \in \Omega$. Given a subspace $V \subset \mathbb{R}^{n}$ then $\Omega:=V^{\perp} \subset \mathbb{R}_{n}^{\perp}$ with $\omega \in \Omega$ if, and only if, for all $s_{i} \in V, \omega s_{i}=0$. I and $\mathbf{0}$ denote respectively the identity and zero matrices of suitable dimensions.

\section{B. Hybrid systems under aperiodic jumps}

Introduce the hybrid time domain $\mathscr{T}=\cup_{k=0}^{\infty}\left[t_{k}, t_{k+1}\right] \times\{k\}$ with $t_{k}-t_{k-1}:=\delta_{k}$ for all $k \in \mathbb{N}$. Accordingly, consider the class of hybrid systems given by

$$
\begin{aligned}
& x^{+}=F x+G v \\
& \dot{x}=A x+B u \\
& y=C x
\end{aligned}
$$

or, more explicitly, for $t \in\left[t_{k}, t_{k+1}[\right.$

$$
\begin{aligned}
& x\left(t_{k}, k\right)=F x\left(t_{k}, k-1\right)+G v(k), \quad \dot{x}(t, k)=A x(t, k)+B u(t, k) \\
& y(t, k)=C x(t, k)
\end{aligned}
$$

with $x \in \mathbb{R}^{n}, u, v, y \in \mathbb{R}^{p}$ and

$$
x\left(t_{k}, k-1\right)=e^{A \delta_{k}} x\left(t_{k-1}, k-1\right)+\int_{t_{k-1}}^{t_{k}} e^{\left(t_{k}-s\right) A} B u(s, k-1) \mathrm{d} s
$$

for $x\left(t_{0},-1\right)=x\left(t_{0}, 0\right)=x_{0}, x\left(t_{k}, k-1\right)=\lim _{t \rightarrow t_{k}^{-}} x(t, k-1)$. In what follows, we denote $C^{\top}=\left(\begin{array}{lll}c_{1}^{\top} & \ldots & c_{p}^{\top}\end{array}\right), B=$ $\left(\begin{array}{lll}b_{1} & \ldots & b_{p}\end{array}\right)$. We assume that the time domain is not known in the sense that measures (or estimates) of the jumping instants are not available. We underline that the class of system (1) under study is also referred to as impulsive systems (e.g., see [16]).

\section{THE HYBRID ZERO-DYNAMICS}

The zero-dynamics of (1) is the residual dynamics the system evolves with when, for a suitable $x_{0} \in \mathbb{R}^{n}$ such that $y_{0}=0$ and a suitable feedback law, one has $y(t, k)=0$ for all $(t, k) \in \mathscr{T}$. From a geometric point of view [14], it is the dynamics governing the evolutions over the largest feedback-unobservable subspace. This is the point of view we shall adopt, so that the following definition of hybrid zero-dynamics will be assumed:

Definition 3.1: The zero-dynamics of (1) is the residual dynamics the system evolves with when the trajectories are constrained onto the zero-dynamics subspace (or, for brevity, zero subspace) $V_{h}^{*} \subset \mathbb{R}^{n}$ that is the largest subspace made unobservable under state feedback.

Accordingly, we shall characterize the zero-dynamics through the definition of the zero subspace $V_{h}^{*}$. As a byproduct, this will lead to a natural interpretation of the zeros of some transfer function associated to (1) as defined, for the periodical case, in [12].

To this end, when considering (1a) and (1b) as purely continuous and discrete-time dynamics with corresponding output (1c) one can define the subspaces $V_{c} \subset \mathbb{R}^{n}$ and $V_{d} \subset \mathbb{R}^{n}$ being the largest invariant subspaces that are, respectively,
$(A, B)$-invariant and $(F, G)$-invariant and contained in $\operatorname{ker} C$; namely, $V_{c}$ and $V_{d}$ verify, separately,

$$
\begin{aligned}
& A V_{c} \subset V_{c}+\operatorname{Im} B \\
& F V_{d} \subset V_{d}+\operatorname{Im} G .
\end{aligned}
$$

with $\operatorname{dim}\left\{V_{c}\right\}=n-r_{c}$ and $\operatorname{dim}\left\{V_{d}\right\}=n-r_{d}$ for some positive real constants $r_{c}, r_{d}$.

Remark 3.1: When the continuous and discrete-time dynamics associated to (1a) and (1b) admit well-defined vector relative degrees $\mathbf{r}_{c}=\left(r_{c}^{1} \ldots r_{c}^{p}\right)$ and $\mathbf{r}_{d}=\left(r_{d}^{1} \ldots r_{d}^{p}\right) \quad[17$, Chapter 5] with $r_{c}=\sum_{i=1}^{p} r_{c}^{i}$ and $r_{d}=\sum_{i=1}^{p} r_{d}^{i}$ [17], $V_{c}$ and $V_{d}$ can be further specified as

$$
V_{c}=\cap_{i=1}^{p} \operatorname{ker}\left(\begin{array}{c}
c_{i} \\
c_{i} A \\
\vdots \\
c_{i} A^{r_{c}^{i}-1}
\end{array}\right), \quad V_{d}=\cap_{i=1}^{p} \operatorname{ker}\left(\begin{array}{c}
c_{i} \\
c_{i} F \\
\vdots \\
c_{i} F^{r_{d}^{i}-1}
\end{array}\right) .
$$

With this in mind, the zero-subspace $V_{h}^{*}$ is thus the largest subspace that is contained in the null-space of $C$ and, at the same time, $(A, B)$ and $(F, G)$-invariant. By definition of $V_{c}$ and $V_{d}$, then one has that necessarily $V_{h}^{*} \subset V_{c} \cap V_{d} \subset \operatorname{ker} C$ so that the following definition can be given.

Theorem 3.1: Consider the hybrid system (1) and the subspaces $V_{c}$ and $V_{d}$ in (3). Denote $V_{\text {int }}:=V_{c} \cap V_{d}$ and define $V_{h}^{*}$ as the largest subspace contained in $V_{\text {int }} \subset \operatorname{ker} C$ verifying

$$
\begin{aligned}
& A V_{h}^{*} \subset V_{h}^{*}+\operatorname{Im} B \\
& F V_{h}^{*} \subset V_{h}^{*}+\operatorname{Im} G
\end{aligned}
$$

with $\operatorname{dim}\left\{V_{h}^{*}\right\}=n-r_{h}$ for some $r_{h} \in \mathbb{N}$. Then, $V_{h}^{*}$ defines the zero-subspace for the hybrid system (1); namely, there exist $K^{*}$ and $H^{*}$ (the friends of $V_{h}^{*}$ ) verifying

$$
\left(A+B K^{*}\right) V_{h}^{*} \subset V_{h}^{*} \quad \text { and } \quad\left(F+G H^{*}\right) V_{h}^{*} \subset V_{h}^{*}
$$

so that, for all $x_{0} \in V_{h}^{*}, y(t, k)=0$ for all $(t, k) \in \mathscr{T}$.

The proof of Theorem 3.1 is quite straightforward. $V_{h}^{*}$ defines the zero-subspace associated to (1) as it is the largest controlled-invariant under both (1a) and (1b) and contained in $\operatorname{ker} C$. In addition, when measures of the jumping times are available, double invariance (4) is no longer necessary for the definition of the zero-subspace. In that case, the necessary and sufficient condition, together with the construction algorithm, has been provided in [15] for controlled invariance of impulsive systems at large. In that case, the friends of $V_{h}^{*}$ under $(A, B)$ and $(F, G)$ depend explicitly on $\delta_{k}$ for all $k \geq 0$.

As a consequence of Theorem 3.1, the zero-dynamics of the hybrid system (1) can be defined as its restriction onto the zero subspace $V_{h}^{*}$, as pointed out in Section III-A below. This results from common characteristics of the underlying geometry of the two possibly different zero-dynamics of $11 \mathrm{a}$ and $(1 \mathrm{~b})$. This aspect is revealed by the proposed state-space approach as it is not evident from the frequency domain characterization proposed in [12].

Remark 3.2: When $p=1$, that is (1) is SISO, then $r_{h}$ can be interpreted as the hybrid relative degree of (1). 
Remark 3.3: When $p=1$ and $V_{h}^{*}=\{0\}$ then $r_{h}=n$ and there is no hybrid zero-dynamics, even if both $V_{c}$ and $V_{d}$ are not $\{0\}$. On the other side, whenever $r_{c}=r_{d}=1$ then $V_{h}^{*}=\operatorname{ker} C$ regardless $A$ and $F$ and, thus, the internal structure of the flow and hybrid dynamics.

In what follows, such a concept will be further clarified by linking the notion of hybrid zeros of (1) to the zeros of the single transfer functions associated to (1a) and (1b). Before doing this, an algorithm for computing $V_{h}^{*}$ is given by extending the one in [13], [14] to this context based on the ones in [15], [18], [19].

\section{A. On the computation of $V_{h}^{*}$}

Let $\Pi_{h}(A, B, F, G, C):=\Pi(A, B, C) \cap \Pi(F, G, C)$ that is the set of subspaces being, at the same time, $(A, B)$ and $(F, G)$ invariant and that are contained in $V_{\text {int }}:=V_{c} \cap V_{d} \subset \operatorname{ker} C$. The set $\Pi_{h}(A, B, F, G, C)$ is closed under subspace addition and ordered and, thus, possesses a supremal element $V_{h}^{*}:=$ $\sup \Pi_{h}(A, B, F, G, C)$ [14, Chapter 4] that can be deduced starting from $V_{c}$ and $V_{d}$ being the maximum elements of, respectively, $\Pi(A, B, C)$ and $\Pi(F, G, C)$ and verifying (3). To this end, the following Lemma is thus useful to characterize all $V \in \Pi_{h}(A, B, F, G, C)$.

Lemma 3.1: Consider the matrices $A, B, F, G, C$ defining the hybrid dynamics $(1)$ and $\Pi_{h}(A, B, F, G, C):=\Pi(A, B, C) \cap$ $\Pi(F, G, C)$. Let $V \subset \mathbb{R}^{n}$ and $\Omega:=V^{\perp} . V \in \Pi_{h}(A, B, F, G, C)$ if and only if

$$
\left(\Omega \cap(\operatorname{Im} B)^{\perp}\right) A+\left(\Omega \cap(\operatorname{Im} G)^{\perp}\right) F \subset \Omega .
$$

Proof: : One needs to show that $(6)$ is equivalent to $(i)$ $V \subset V_{\text {int }} \subset \operatorname{ker} C$; (ii) $A V \subset V+\operatorname{Im} B$; (iii) $F V \subset V+\operatorname{Im} G$. In due dual space, one gets that $V$ verifies $(i),(i i)$ and (iii), if and only if $\Omega$ verifies: (ib) ( $\operatorname{ker} C)^{\perp} \subset\left(V_{\text {int }}\right)^{\perp} \subset \Omega$; (iib) $(\Omega \cap$ $\left.(\operatorname{Im} B)^{\perp}\right) A \subset \Omega ;(i i i b)\left(\Omega \cap(\operatorname{Im} G)^{\perp}\right) F \subset \Omega$. In particular, (ii) and (iii) hold true at the same time if, and only if (iib) and (iiib) do and, as a consequence, (6) holds true. As a matter of fact, $\forall \omega_{1} \in \Omega \cap(\operatorname{Im} B)^{\perp}$ and $\forall \omega_{2} \in \Omega \cap(\operatorname{Im} G)^{\perp}$ one has $\omega_{1} A v=0$ and $\omega_{2} F v=0$ for all $v \in V$ if, and only if, $\forall \omega_{1} A \in\left(\Omega \cap(\operatorname{Im} B)^{\perp}\right)$ and $\forall \omega_{2} F \in\left(\Omega \cap(\operatorname{Im} G)^{\perp}\right)$ that is $\omega_{1} A \in \Omega$ and $\omega_{2} F \in \Omega$. Setting now $\omega=\omega_{1} A+\omega_{2} F, \omega \in$ $\left(\Omega \cap(\operatorname{Im} B)^{\perp}\right) A+\left(\Omega \cap(\operatorname{Im} G)^{\perp}\right) F$ and $\omega \in \Omega$ holding if and only if (6) does.

Starting from Lemma 3.1, the next result allows to construct the maximal $(A, B)$ and $(F, G)$ invariant subspace $V_{h}^{*}$ that is contained in $V_{\text {int }} \subset \operatorname{ker} C$ and that defines the zerosubspace of the hybrid system. As typical in the geometric approach, $V_{h}^{*}$ is deduced in the dual space by defining the minimal dimension co-subspace $\Omega^{*}$ verifying Lemma 3.1 .

Theorem 3.2: Consider the hybrid dynamics (1) and $\Pi_{h}(A, B, F, G, C):=\Pi(A, B, C) \cap \Pi(F, G, C)$ with $V_{\text {int }}:=V_{c} \cap$ $V_{d} \subset \operatorname{ker} C$ and $V_{c}$ and $V_{d}$ as in (3). Introduce the sequence $\left\{\Omega^{\ell}\right\}$ as $\Omega^{0}=V_{\text {int }}^{\perp}$ and

$$
\Omega^{\ell}=V_{\text {int }}^{\perp}+\left(\Omega^{\ell-1} \cap(\operatorname{Im} B)^{\perp}\right) A+\left(\Omega^{\ell-1} \cap(\operatorname{Im} G)^{\perp}\right) F
$$

with $\ell=1,2, \ldots$ Then, $\Omega^{\ell} \subset \Omega^{\ell-1}$ and, for some $\ell \geq \ell^{*}$ with $\ell^{*} \leq \operatorname{dim}\left(V_{\text {int }}\right), \Omega^{*}=\Omega^{\ell}=\Omega^{\ell+1}$. As a consequence, the zero-subspace $V_{h}^{*}$ is given by

$$
V_{h}^{*}:=\left(\Omega^{*}\right)^{\perp} .
$$

Proof: By construction, one gets $\Omega^{0} \subset \Omega^{1} \subset \cdots \subset \Omega^{\ell}$ for $\ell=1,2, \ldots$. Thus, there exists a $\ell^{*} \leq \operatorname{dim}\left(V_{\text {int }}\right)$ such that $\Omega^{\ell}=\Omega^{\ell+1}$ for all $\ell \geq \ell^{*}$. Moreover, from Lemma 3.1. $V_{h}^{*}=\left(\Omega^{*}\right)^{\perp} \in \Pi_{h}(A, B, F, G, C)$ and, by construction, $V_{h}^{*}=\sup \Pi_{h}(A, B, F, G, C)$.

Remark 3.4: Theorems 3.1 and 3.2 (and all the results to come) extend to the case in which the output mappings are switching between flows and jumps, that is to systems of the form (1) where (1c) is modified as follows

$$
y(t, k)= \begin{cases}C_{f} x(t, k-1) & \text { if } t \in\left[t_{k-1}, t_{k}\right) \\ C_{j} x\left(t_{k}, k-1\right) & \text { if } t=t_{k} .\end{cases}
$$

In that case, one computes $V_{c}$ and $V_{d}$ as the subspaces associated to $\left(A, B, C_{f}\right)$ and $\left(F, G, C_{j}\right)$ and proceeds along the same lines.

Remark 3.5: When $G=0$, the proposed algorithm recovers the one presented in [18] for disturbance decoupling under periodic jumps. In addition, it represents an alternative to the one settled in [19] for regulation of aperiodically jumping hybrid systems.

\section{B. An invariance-based decomposition}

From Theorem 3.1 $\operatorname{dim}\left\{V_{h}^{*}\right\}=n-r_{h}$ so that, from Theorem 3.2. $\operatorname{dim}\left\{\Omega^{*}\right\}=r_{h}$. As a consequence, we rewrite $\Omega^{*}=\operatorname{span}\left\{\omega_{1}, \ldots, \omega_{r_{h}}\right\}$ with $\omega_{i}$ being row vectors verifying, for all $s \in V_{h}^{*}$ that $\omega_{i} s=0$ for $i=1, \ldots, r_{h}$. Introduce now the coordinate transformation

$$
\left(\frac{z}{\eta}\right)=T x, \quad T:=\left(\begin{array}{llll}
\omega_{1}^{\top} & \ldots & \omega_{r_{h}}^{\top} & T_{2}^{\top}
\end{array}\right)^{\top}
$$

with $T_{2} \in \operatorname{Mat}_{\mathbb{R}}\left(n-r_{h}, n\right)$ being a complement so that $|T| \neq 0$ and $\omega$ and $z \in \mathbb{R}^{r_{h}}$ and $\eta \in \mathbb{R}^{n-r_{h}}$. Then, one gets

$$
\begin{array}{ll}
T A T^{-1}=\left(\begin{array}{ll}
A_{11} & A_{12} \\
A_{21} & A_{22}
\end{array}\right), & T B=\left(\begin{array}{l}
B_{1} \\
B_{2}
\end{array}\right) \\
T F T^{-1}=\left(\begin{array}{ll}
F_{11} & F_{12} \\
F_{21} & F_{22}
\end{array}\right), \quad T G=\left(\begin{array}{l}
G_{1} \\
G_{2}
\end{array}\right), \quad C T^{-1}=\left(\begin{array}{ll}
C_{1} & \mathbf{0}
\end{array}\right)
\end{array}
$$

with $\operatorname{Im} A_{12} \subset \operatorname{Im} B_{1}$ and $\operatorname{Im} F_{12} \subset \operatorname{Im} G_{1}$. Thus, in these new coordinates, the friends of $V_{h}^{*}$ under $(A, B)$ and $(F, G)$ are of the form $K^{*}=\left(\begin{array}{ll}0 K_{r}^{*}\end{array}\right)$ and $H^{*}=\left(\begin{array}{ll}0 H_{r}^{*}\end{array}\right)$ and ensure

$$
A_{12}+B_{1} K_{r}^{*}=0, \quad F_{12}+G_{1} H_{r}^{*}=0 .
$$

so that, when setting $K_{r}^{*}=-\left(B_{1}^{\top} B_{1}\right)^{-1} B_{1}^{\top} A_{12}, \quad H_{r}^{*}=$ $-\left(G_{1}^{\top} G_{1}\right)^{-1} G_{1}^{\top} F_{12}$ and

$$
u^{*}=\bar{u}+K_{r}^{*} \eta, \quad v^{*}=\bar{v}+H_{r}^{*} \eta
$$

the dynamics 11 get the form

$$
\begin{aligned}
& z^{+}=F_{11} z+G_{1} \bar{v} \\
& \eta^{+}=F_{21} z+\left(F_{22}+G_{2} H_{r}^{*}\right) \eta+G_{2} \bar{v} \\
& \dot{z}=A_{11} z+B_{1} \bar{u} \\
& \dot{\eta}=A_{21} z+\left(A_{22}+B_{2} K_{r}^{*}\right) \eta+B_{2} \bar{u} \\
& y=C_{1} z
\end{aligned}
$$


From the previous representation, it is clear that when $x \in V_{h}^{*}$, then $z=0$ so that the residual dynamics governing (1) are

$$
\eta^{+}=Q_{d} \eta, \quad \dot{\eta}=Q_{c} \eta
$$

with $Q_{c}:=F_{22}+G_{2} H_{r}^{*}$ and $Q_{d}=A_{22}+B_{2} K_{r}^{*}$. The hybrid system (13) describes the hybrid zero-dynamics over $V_{h}^{*}$.

The form (12) underlines that the feedback (11) is the one generating maximal unobservability of (1) by making the subspace $V_{h}^{*}$ defined in Theorem 3.1 unobservable.

Remark 3.6: Contrarily to previous results for hybrid systems (e.g., [8]), thanks to the geometric characterization, the feedback laws (11) rendering the zero-dynamics invariant do not require explicit computation of the trajectories of (1) as they only depend on the matrices $A, B, F, G, C$ and the properties they yield. In addition, the knowledge of the jumping period sequence $\left\{\delta_{0}, \delta_{1}, \ldots\right\}$ is not required.

Accordingly, the following definition is straightforward.

Definition 3.2 (Minimum-phase of hybrid LTI systems):

The hybrid system (1) is said to be minimum-phase when the zero-dynamics (13) are asymptotically stable.

Conditions for investigating the stability of (13) are not given as beyond the purpose of the paper. However, the reader is referred to several references on the topics for sufficient conditions and a deeper understanding on the difficulties (e.g., [20], [21] and references therein).

In what follows, further comments on the characterization of $Q_{c}$ and $Q_{d}$ are discussed with special emphasis on their relations with the zeros of the transfer functions involved.

\section{INSIGHTS TO THE HYBRID ZERO DYNAMICS}

\section{A. Jumping and flowing zeros}

Unless differently specified and for the sake of simplicity, let (1) be a SISO system with $u, v, y \in \mathbb{R}$. Assume the couples $(A, B)$ and $(F, G)$ controllable and $(A, C)$ and $(F, C)$ observable. Consider now the minimal transfer functions associated with the individual flow and jump dynamics (1a) and $(1 \mathrm{~b})$ when considered as purely continuous and discretetime systems

$$
\begin{aligned}
& P(s)=C(s \mathbf{I}-A)^{-1} B=\frac{b_{0}+\cdots+b_{n-r_{c}} s^{n-r_{c}}}{a_{0}+\cdots+a_{n-1} s^{n-1}+s^{n}} \\
& L(s)=C(s \mathbf{I}-F)^{-1} G=\frac{g_{0}+\cdots+g_{n-r_{d}} s^{n-r_{d}}}{f_{0}+\cdots+f_{n-1} s^{n-1}+s^{n}}
\end{aligned}
$$

with $s \in \mathbb{C}$, possessing, respectively, $n-r_{c}$ and $n-r_{d}$ zeros defined by the roots of the numerators of the corresponding transfer function. The next result shows the relation among the zeros of $P(s)$ and $L(s)$ with the $r_{h}$ eigenvalues of $Q_{c}$ and the $r_{h}$ eigenvalues of $Q_{d}$ as given in (13). For the sake of compactness denote by $Z_{c}$ and $Z_{d}$, respectively, the zeros of $P(s)$ and $L(s)$ that is

$$
\begin{aligned}
& Z_{c}=\left\{s \in \mathbb{C} \text { s.t. } b_{0}+b_{1} s+\cdots+b_{n-r_{c}} s^{n-r_{c}}=0\right\} \\
& Z_{d}=\left\{s \in \mathbb{C} \text { s.t. } g_{0}+g_{1} s+\cdots+g_{n-r_{d}} s^{n-r_{d}}=0\right\}
\end{aligned}
$$

and referred to as the sets of flowing and jumping zeros. We shall refer to $s_{i} \in \sigma\left(Q_{c}\right)$ and $z_{j} \in \sigma\left(Q_{d}\right)$ as, respectively, the hybrid-flowing and hybrid-jumping zeros.
Theorem 4.1: Consider the hybrid system (11) with $p=1$ and zero-dynamics of dimension $n-r_{h}$ evolving as (13) over the zero-subspace $V_{h}^{*}$. Consider the transfer functions (14) and the corresponding flowing and jumping zeros in $Z_{c}$ and $Z_{d}$. Then, the following inclusions hold true

$$
\begin{aligned}
& \sigma\left(Q_{c}\right) \subset Z_{c} \\
& \sigma\left(Q_{d}\right) \subset Z_{d} .
\end{aligned}
$$

Proof: The proof is given only for $(15 \mathrm{~b})$ as it follows the same lines for the flow dynamics. We first recall from [17] that, given matrices $(F, G, C)$ then the zeros $Z_{d}$ are given by the roots of the polynomial

$$
\left|\left(\begin{array}{cc}
F-s \mathrm{I} & G \\
C & 0
\end{array}\right)\right|=g_{0}+g_{1} s+\cdots+g_{n-r_{d}} s^{n-r_{d}} .
$$

The above polynomial is invariant under feedback and coordinate transformations so that introducing $T$ and $H^{*}$ as in (97)-(11) and $Q_{d}=F_{22}+G_{2} H^{*}$ one has

$$
\left|\left(\begin{array}{cc}
F-s \mathrm{I} & G \\
C & 0
\end{array}\right)\right|=\left|\left(\begin{array}{cc}
F_{11}-s \mathrm{I} & G_{1} \\
C_{1} & 0
\end{array}\right)\right|\left|Q_{d}-s \mathrm{I}\right| .
$$

Now, by applying the Schur complement, one gets

$$
\left.\left|\left(\begin{array}{cc}
F_{11}-s \mathrm{I} & G_{1} \\
C_{1} & 0
\end{array}\right)\right|=\left|F_{11}-s \mathrm{I}\right| \mid C_{1}\left(s \mathrm{I}-F_{11}\right)^{-1} G_{1}\right) \mid .
$$

The polynomial $\left|F_{11}-s \mathrm{I}\right|$ defines the $r_{h}$ eigenvalues of the matrix $F_{11}$ whereas, in the SISO case $\operatorname{det}\left(C_{1}\left(s \mathrm{I}-F_{11}\right)^{-1} G_{1}\right)$ is the transfer function associated to $(F, G, C)$ under the feedback $H^{*}$ so that

$$
\left|\left(C_{1}\left(s \mathrm{I}-F_{11}\right)^{-1} G_{1}\right)\right|=\frac{\bar{g}_{0}+\bar{g}_{1} s+\cdots+\bar{g}_{\bar{m}} s^{\bar{m}}}{\operatorname{det}\left(F_{11}-s \mathrm{I}\right)}
$$

with the numerator defining the zeros with $\bar{m}<r_{h}$. Thus, by plugging now the above relation into (17) one gets

$$
\left|\left(\begin{array}{cc}
F-s \mathrm{I} & G \\
C & 0
\end{array}\right)\right|=\left(\bar{g}_{0}+\bar{g}_{1} s+\cdots+\bar{g}_{\bar{m}} s^{\bar{m}}\right)\left|Q_{d}-s \mathrm{I}\right| .
$$

Equating the right-hand sides of (16) and (18) one gets

$g_{0}+g_{1} s+\cdots+g_{n-r_{d}} s^{n-r_{d}}=\left|Q_{d}-s \mathrm{I}\right|\left(\bar{g}_{0}+\bar{g}_{1} s+\cdots+\bar{g}_{\bar{m}} s^{\bar{m}}\right)$ so that necessarily, $\bar{m}=r_{h}-r_{d}$ with $\left|Q_{d}-s \mathrm{I}\right|$ being a factor of the polynomial identifying the zeros associated to $(F, G, C)$. Thus, one gets that $\sigma\left(Q_{d}\right) \subset Z_{d}$.

It is worth to note that invariance of $V_{h}^{*}$ (that is unobservability) under (1) is yielded under partial zero-cancelation that is by erasing the zeros of $P(s)$ and $L(s)$ in 114 making the jump and flow behaviour over $V_{h}^{*}$ compatible. As a consequence, one does not need $Z_{c} \cap Z_{d} \neq \varnothing$ as (13) does not depend on the actual values of the zeros of (1a) and (1b) but on the common subspaces induced by the hybrid interconnection. Also, the definition of the hybrid zerodynamics is independent on the poles and eigenvalues of the matrices $A$ and $F$.

Remark 4.1: Those arguments extend to the MIMO case by noticing that the numerator of

$$
\left|C_{1}\left(s \mathrm{I}-F_{11}\right)^{-1} G_{1}\right|=\frac{\bar{g}_{0}+\bar{g}_{1} s+\cdots+\bar{g}_{\bar{m}} s^{\bar{m}}}{\operatorname{det}\left(F_{11}-s \mathrm{I}\right)}
$$


defines the closed-loop transmission zeros with $\bar{m}<r_{h}$. In that case, $\sigma\left(Q_{c}\right)$ and $\sigma\left(Q_{d}\right)$ define the $n-r_{h}$ dimensional subset of the transmission zeros associated to, respectively, $(A, B, C)$ and $(F, G, C)$.

In this context, one can re-define the zero-dynamics via the definition of a suitable exosystem whose series interconnection with (1) generates an identically zero output evolution, under suitable initial condition. The following result is given by extending the usual definition of zeros (e.g., [17]).

Proposition 4.1: Let the hybrid subsystem (1) possess the zero-subspace $V_{h}^{*}$ defined as in Theorem 3.1. Consider the exosystem $\xi^{+}=Q_{d} \xi, \dot{\xi}=Q_{c} \xi$ with $\xi \in \mathbb{R}^{n-r_{h}}$ and interconnected to 11 through $u=K_{r}^{*} \xi$ and $v=H_{r}^{*} \xi$ as in 10. Then, for all $x_{0} \in V_{h}^{*}$, there exists $\xi_{0} \in \mathbb{R}^{n-r_{h}}$ such that $y(t, k)=0$ for all $(t, k) \in \mathscr{T}$. More in details, this is given by $\xi_{0}=T_{2} x_{0}$ with $T_{2} \in \operatorname{Mat}_{\mathbb{R}}\left(n-r_{h}, n\right)$ defined as in 9 .

\section{B. On the hybrid zero-sets}

The results stated so far put in light that a notion of zerodynamics can be settled in the hybrid context: roughly speaking such a notion is related to the maximal subspace, $V_{h}^{*}$, which can be rendered unobservable for both the flowing and jumping dynamics under suitable state feedbacks $u=H^{*} x$ and $v=K^{*} x$. When constrained over such a subspace the hybrid dynamics evolve according to continuous and discretetime behaviors associated to $\sigma\left(Q_{c}\right) \subset Z_{c}$ and $\sigma\left(Q_{d}\right) \subset Z_{d}$. What is peculiar of these subsets of zeros which have the same cardinality, is that they share the maximal unobservable subspace under feedbacks. With this in mind, the definition of zeros-set links the notion of hybrid zero-dynamics to the zeros of the involved transfer functions.

Definition 4.1: The zeros-set of the hybrid system (1) is defined as $Z_{h}^{*}=\sigma\left(Q_{c}\right) \times \sigma\left(Q_{d}\right) \subset Z_{c} \times Z_{d}$.

It must be noted that such notion of zeros-set is valid for any system resulting from the interlink of different LTI controlled continuous-time and/or discrete-time dynamics defined over the same state space $X \subset \mathbb{R}^{n}$, with the same output $y=C x$.

Definition 4.1 extends to the hybrid context the notion of the set of zeros of a given transfer function. It is worth to note that the equivalent notion of zero of a transfer function does not have in general a hybrid counterpart since, for a fixed pair $\left(s_{k}, z_{k}\right) \in Z_{h}^{*}$, as any transfer function's zero does, the existence of a one-dimensional subspace which can be rendered unobservable under feedback is not guaranteed.

The discussion about the possibility of computing zerossubsets of the zeros-set $Z_{h}^{*}$ (not developed here for the sake of space) can be deepened starting from these simple elements and the understanding is left to two elementary examples in the sequel. Assuming that a given hybrid system has a zerosset of cardinality at least two, the presence of zeros-subsets corresponds to the existence of feedback-unobservable subspaces shared by the flowing and jumping dynamics of dimensions less then $\left(n-r_{h}\right)$ (that is the maximal one); in doing this, one takes into account that unobservability can be generated only by cancelling couples of zeros in $Z_{h}^{*}$.

\section{SOME ILLUSTRATING EXAMPLES}

\section{A. Example 1}

Consider the hybrid system (1) with

$A=\left(\begin{array}{lll}0 & 1 & 0 \\ 0 & 0 & 1 \\ 0 & 0 & 0\end{array}\right), B=\left(\begin{array}{l}0 \\ 0 \\ 1\end{array}\right), F=\left(\begin{array}{lll}1 & 1 & 0 \\ 0 & 1 & 1 \\ 0 & 0 & 1\end{array}\right), G=\left(\begin{array}{c}1 \\ 0 \\ -1\end{array}\right)$

and $C=\left(\begin{array}{lll}-1 & 0 & 1\end{array}\right)$. Since $r_{c}=r_{d}=1$ then $r_{h}=1$ and

$$
V_{h}^{*}=V_{c}=V_{d}=\operatorname{span}\left\{\left(\begin{array}{lll}
1 & 0 & 1
\end{array}\right)^{\top},\left(\begin{array}{lll}
0 & 1 & 0
\end{array}\right)^{\top}\right\} .
$$

Thus, the hybrid zero-dynamics 13 are characterized by

$$
Q_{c}=\left(\begin{array}{ll}
0 & 1 \\
1 & 0
\end{array}\right), \quad Q_{d}=\left(\begin{array}{cc}
1 & \frac{1}{2} \\
1 & 1
\end{array}\right)
$$

Then the hybrid zeros-set results to be $Z_{h}^{*}=\{(-1,1)\} \times$ $\left\{1-\frac{\sqrt{2}}{2}, 1+\frac{\sqrt{2}}{2}\right\}$ with corresponding eigenvectors, under the coordinate transformation above, $w_{1}^{f}=\left(\begin{array}{lll}0 & 1 & -1\end{array}\right)^{\top}, w_{2}^{f}=$ $\left(\begin{array}{lll}0 & 1 & 1\end{array}\right)^{\top}, w_{1}^{j}=\left(\begin{array}{lll}0 & 1 & -\sqrt{2}\end{array}\right)^{\top}, w_{2}^{j}=\left(\begin{array}{lll}0 & 1 & \sqrt{2}\end{array}\right)^{\top}$. From this computation, $V_{h}^{*}$ is the unique subspace that is $(A, B)$ and $(F, G)$ invariant so that $Z_{h}^{*}$ of cardinality two is the zeros-set and no zeros-subset exists.

\section{B. Example 2}

Consider the hybrid system (1) with $A$ and $F$ as in Example $\mathrm{V}-\mathrm{A}$ and

$$
B=\left(\begin{array}{l}
0 \\
0 \\
1
\end{array}\right), \quad G=\left(\begin{array}{c}
1 \\
0 \\
\frac{\sqrt{3}-3}{6}
\end{array}\right), \quad C=\left(\begin{array}{lll}
\frac{\sqrt{3}-3}{6} & \frac{\sqrt{3}+3}{6} & 1
\end{array}\right) .
$$

As in the previous example, $r_{h}=r_{c}=r_{d}=1$ and

$$
V_{h}^{*}=V_{c}=V_{d}=\operatorname{span}\left\{\left(\begin{array}{lll}
1 & 0 & \frac{3-\sqrt{3}}{6}
\end{array}\right)^{\top},\left(\begin{array}{lll}
0 & 1 & -\frac{\sqrt{3}+3}{6}
\end{array}\right)^{\top}\right\} .
$$

In that case, one gets the hybrid zero-dynamics (13) with

$$
Q_{c}=\left(\begin{array}{cc}
0 & 1 \\
\frac{3-\sqrt{3}}{6} & -\frac{3+\sqrt{3}}{6}
\end{array}\right), \quad Q_{d}=\left(\begin{array}{cc}
\frac{\sqrt{3}+15}{4} & -\frac{5 \sqrt{3}-3}{12} \\
\frac{3-\sqrt{3}}{6} & \frac{3-\sqrt{3}}{6}
\end{array}\right)
$$

so that the zeros-set is given by $Z_{h}^{*}=\left\{-1, \frac{\sqrt{3}-3}{6}\right\} \times$ $\left\{\frac{3-\sqrt{3}}{3}, \frac{3+\sqrt{3}}{4}\right\}$. By computing the corresponding eigenvectors, in this case the same eigenvector is associated to a pair of a continuous-time and a discrete-time eigenvalues, namely $w_{2}^{j}=\left(\begin{array}{lll}0 & \frac{3 \sqrt{3}+4}{2} & 1\end{array}\right)^{\top}, w_{1}^{f}=w_{1}^{j}=\left(\begin{array}{lll}0 & 1 & 1\end{array}\right)^{\top}$ and $w_{2}^{f}=\left(\begin{array}{lll}0 & 1 & \frac{2-\sqrt{3}}{6}\end{array}\right)^{\top}$. Since $s_{1}=-1$ and $z_{1}=\frac{3-\sqrt{3}}{3}$ share a one dimensional invariant subspace $\operatorname{span}\left\{w_{1}^{f}\right\}$ (that is an $(A, B)$ and $(F, G)$ invariant subspace in kerC), the system also possesses the hybrid zeros-subset $z_{h}=\left\{\left(-1, \frac{3-\sqrt{3}}{3}\right)\right\}$ of cardinality one; it should be assumed to define a zero-pair as the counterpart of the zero for classical systems. 


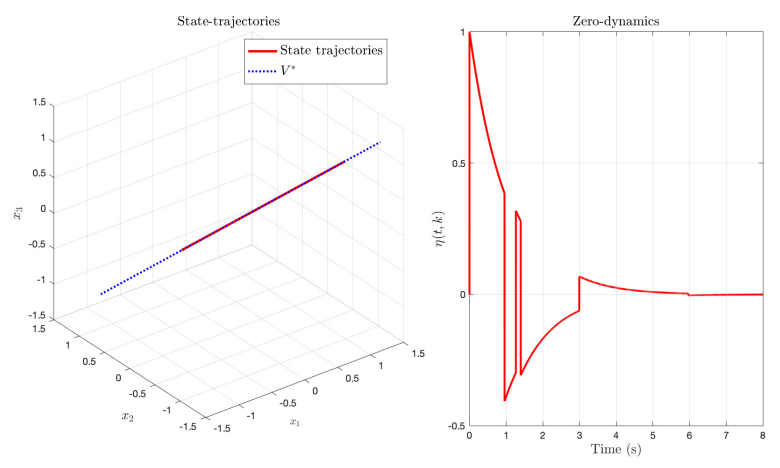

Fig. 1. $\quad x_{0} \in V_{h}^{*}$ and $y(t, k)=0$

\section{Example 3}

Consider the simple example deduced by (1) when

$$
\begin{aligned}
& A=\left(\begin{array}{lll}
0 & 1 & 0 \\
0 & 0 & 1 \\
0 & 0 & 0
\end{array}\right), \quad B=\left(\begin{array}{l}
0 \\
0 \\
1
\end{array}\right), \quad C=\left(\begin{array}{lll}
2 & 3 & 1
\end{array}\right) \\
& F=\left(\begin{array}{ccc}
-\frac{1}{9} & \frac{7}{12} & -\frac{17}{36} \\
-\frac{4}{3} & -1 & \frac{4}{3} \\
-\frac{1}{9} & \frac{1}{3} & -\frac{2}{9}
\end{array}\right), \quad G=\left(\begin{array}{c}
1 \\
0 \\
-2
\end{array}\right) .
\end{aligned}
$$

From Theorem 3.1, the zero-subspace is

$$
V_{h}^{*}=V_{d}=\operatorname{span}\left(\begin{array}{c}
1 \\
-1 \\
1
\end{array}\right) \subset V_{c}=\operatorname{span}\left\{\left(\begin{array}{c}
1 \\
0 \\
-2
\end{array}\right),\left(\begin{array}{c}
0 \\
1 \\
-3
\end{array}\right)\right\}
$$

that is, at the same time, $(A, B)$ and $(F, G)$-invariant. Thus, $r_{h}=2=\max \left\{r_{c}, r_{d}\right\}$ with $r_{c}=1, r_{d}=2, Z_{c}=\{-1,-2\}$ and $\left.Z_{d}=\{-1\}\right)$. By specifying (9) with $T_{2}=\left(\begin{array}{lll}0 & 1 & 0\end{array}\right), \omega_{1}=C$ and $\omega_{2}=C F$, one gets $C T^{-1}=\left(\begin{array}{lll}1 & 0 & 0\end{array}\right)$

$$
\begin{aligned}
& T F T^{-1}=\left(\begin{array}{ccc}
0 & 1 & 0 \\
\frac{13}{36} & -\frac{1}{3} & -\frac{5}{3} \\
\frac{1}{5} & \frac{2}{5} & -1
\end{array}\right), \quad T G=\left(\begin{array}{c}
0 \\
-10 \\
0
\end{array}\right) \\
& T A T^{-1}=\left(\begin{array}{ccc}
\frac{13}{10} & \frac{3}{5} & -1 \\
-\frac{13}{20} & -\frac{3}{10} & -\frac{17}{6} \\
\frac{13}{30} & \frac{1}{5} & -1
\end{array}\right), \quad T B=\left(\begin{array}{c}
1 \\
\frac{17}{6} \\
0
\end{array}\right) .
\end{aligned}
$$

The friends 11 of $V_{h}^{*}$ are given by $H^{*}=\left(\begin{array}{lll}0 & 0 & -\frac{1}{6}\end{array}\right)$ and $K^{*}=\left(\begin{array}{lll}0 & 0 & -1\end{array}\right)$ so getting that the zero-dynamics (13) evolve with $Q_{c}=-1$ and $Q_{d}=-1$. The hybrid system is minimum-phase as long as $\delta_{k}>0$ for all $k \geq 0$ as the zero-dynamics is scalar. The zero-set $Z_{h}^{*}$ is given by the only pair $\{(-1,-1)\}$ (composed by flowing and jumping zeros incidentally coincident) which is the zero-pair. For completeness, a simulation is in Figure 1 when assuming a random sequence of jumping times and $x_{0}=\left(\begin{array}{lll}1 & -1 & 1\end{array}\right)^{\top} \in V_{h}^{*}$.

\section{Conclusions And PERSPeCtives}

In this paper, the notion of zero-dynamics has been characterized for classes of linear time invariant hybrid systems under aperiodic time-driven jumps. Following works as [15], [22], the geometric framework contributes to a better understanding on the zero dynamics of hybrid linear systems under aperiodic jumps. Current work is toward the extension to the nonlinear context and the definition of a weaker notion of zero-dynamics revealed when constraining the output to zero only at the jumping instants as emblematic for the sampling zeros of aperiodic sampled dynamics.

\section{ACKNOWLEDGMENTS}

The authors are deeply in debt with the anonymous reviewers for the valuable comments and suggestions.

\section{REFERENCES}

[1] R. Goebel, R. G. Sanfelice, and A. R. Teel, Hybrid Dynamical Systems: modeling, stability, and robustness. Princeton University Press, 2012

[2] F. Ferrante and Y. Wang, "Robust almost global splay state stabilization of pulse coupled oscillators," IEEE Transactions on Automatic Control, vol. 62, no. 6, pp. 3083-3090, 2017.

[3] L. Marconi and A. R. Teel, "Internal model principle for linear systems with periodic state jumps," IEEE Transactions on Automatic Control, vol. 58, no. 11, pp. 2788-2802, 2013.

[4] A. Sferlazza, S. Tarbouriech, and L. Zaccarian, "Time-varying sampled-data observer with asynchronous measurements," IEEE Transactions on Automatic Control, vol. 64, no. 2, pp. 869-876, 2019.

[5] M. Zhang and T.-J. Tarn, "Hybrid control of the pendubot," IEEE/ASME transactions on mechatronics, vol. 7, no. 1, pp. 79-86, 2002.

[6] J. W. Grizzle, C. Chevallereau, R. W. Sinnet, and A. D. Ames, "Models, feedback control, and open problems of 3d bipedal robotic walking," Automatica, vol. 50, no. 8, pp. 1955-1988, 2014.

[7] L. Marconi and A. R. Teel, "A note about hybrid linear regulation," in Decision and Control (CDC), 49th IEEE Conference on, 2010.

[8] D. Carnevale, S. Galeani, and L. Menini, "Output regulation for a class of linear hybrid systems. part 1: trajectory generation," in Decision and Control (CDC), IEEE 51st Annual Conference on, 2012.

[9] E. R. Westervelt, J. W. Grizzle, and D. E. Koditschek, "Hybrid zero dynamics of planar biped walkers," IEEE transactions on automatic control, vol. 48, no. 1, pp. 42-56, 2003.

[10] C. Cai, R. Goebel, R. G. Sanfelice, and A. R. Teel, "Hybrid systems: limit sets and zero dynamics with a view toward output regulation," in Analysis and Design of Nonlinear control systems. Springer, 2008, pp. 241-261.

[11] A. D. Ames, K. Galloway, K. Sreenath, and J. W. Grizzle, "Rapidly exponentially stabilizing control lyapunov functions and hybrid zero dynamics," IEEE Transactions on Automatic Control, vol. 59, no. 4, pp. 876-891, 2014.

[12] S. Galeani, C. Possieri, and M. Sassano, "Zeros and poles of transfer functions for linear hybrid systems with periodic jumps," in Decision and Control (CDC), 2017 IEEE 56th Annual Conference on. IEEE, 2017, pp. 5469-5474.

[13] W. M. Wonham, "Linear multivariable control," in Optimal control theory and its applications. Springer, 1974, pp. 392-424.

[14] G. Basile and G. Marro, Controlled and conditioned invariants in linear system theory. Prentice Hall Englewood Cliffs, 1992.

[15] D. A. Lawrence, "Controlled invariant subspaces for linear impulsive systems," in American Control Conference, 2014, pp. 2336-2341.

[16] E. Zattoni, A. M. Perdon, and G. Conte, "Measurement dynamic feedback output regulation in hybrid linear systems with state jumps," International Journal of Robust and Nonlinear Control, vol. 28, no. 2, pp. 416-436, 2018.

[17] A. Isidori, Nonlinear Control Systems. Springer-Verlag, 1995.

[18] G. Conte, A. M. Perdon, and E. Zattoni, "The disturbance decoupling problem for jumping hybrid systems," in 2015 54th IEEE Conference on Decision and Control (CDC). IEEE, 2015, pp. 1589-1594.

[19] D. Carnevale, S. Galeani, L. Menini, and M. Sassano, "Output regulation of hybrid linear systems with unpredictable jumps," IFAC Proceedings Volumes, vol. 47, no. 3, pp. 1531-1536, 2014.

[20] F. Blanchini and S. Miani, Set-theoretic methods in control. Springer, 2008

[21] G. Chesi, "Lmi conditions for time-varying uncertain systems can be non-conservative," Automatica, vol. 47, no. 3, pp. 621-624, 2011.

[22] E. Zattoni, A. M. Perdon, and G. Conte, "Output regulation by error dynamic feedback in hybrid systems with periodic state jumps," Automatica, vol. 81, pp. 322-334, 2017. 